DAMTP 96-111

\title{
First Order Vortex Dynamics
}

\author{
N.S. Manton* \\ Department of Applied Mathematics and Theoretical Physics \\ University of Cambridge \\ Silver Street, Cambridge CB3 9EW, England
}

\begin{abstract}
A non-dissipative model for vortex motion in thin superconductors is considered. The Lagrangian is a Galilean invariant version of the Ginzburg-Landau model for time-dependent fields, with kinetic terms linear in the first time derivatives of the fields. It is shown how, for certain values of the coupling constants, the field dynamics can be reduced to first order differential equations for the vortex positions. Two vortices circle around one another at constant speed and separation in this model.
\end{abstract}

* email: N.S.Manton@damtp.cam.ac.uk 


\section{Introduction}

Magnetic flux penetrates a Type II superconductor in the form of vortices [1], and recently it has become possible to produce images of vortices sufficiently rapidly that their motion can be observed directly [2]. In the Ginzburg-Landau theory of superconductivity, a charged scalar field representing the electron-pair condensate is coupled to the electromagnetic field. The basic vortex solution, discovered by Abrikosov [3], is a localised magnetic flux tube surrounded by a circulating supercurrent.

The Ginzburg-Landau potential energy functional contains only one dimensionless coupling constant $\lambda$. The value $\lambda=1$ (in our units) is mathematically particularly interesting, because in this case there are no forces between static vortices, and there is a continuous family of static multivortex solutions. A Type II superconductor is modelled by $\lambda>1$. In this case, the potential energy of a two-vortex configuration decreases as the separation increases, in other words, vortices repel [4]. However, there are several possibilities for how the vortices might move, depending on the nature of the dynamical equations for the fields. Let us ignore pinning, which tends to prevent vortex motion at all. The first possibility is that the vortex acceleration is proportional to the force acting. This is what occurs in the relativistic generalisation of the Ginzburg-Landau model, known as the Abelian Higgs model. Relativistic vortices may be interpreted as a solitonic version of fundamental strings [5], or as strings joining confined quarks, or as cosmic strings produced at a phase transition early in the universe's history [6]. The second possibility is that the vortex velocity is proportional to the force. This is modelled by dissipative equations involving the first time derivatives of the fields [7]. Recently, one version of such equations, the Ginzburg-Landau gradient flow equations, have been analysed in detail [8]. The third possibility is that the vortex motion is at right angles to the force, so two vortices circulate around each other, as in a fluid, and there is no dissipation [9]. In real superconductors, vortex motion is usually dissipative, but at very low temperatures, it has been argued that the third type of motion would occur [10].

The response of vortices to an applied electric "transport" current, perpendicular to the vortex cores, can distinguish the second and third types of motion. If the vortices move at right angles to the current, then the dynamics is dissipative, but if they are carried along by the current (again, as in a fluid) then the dynamics is non-dissipative. To see this, note that a moving vortex has an electric field in its core, perpendicular to both the velocity and the direction of the magnetic flux. Also, part of the applied current penetrates the vortex core, where it becomes a normal electric current. There is dissipation when the 
current and electric field in the core are parallel, but not when they are perpendicular.

The purpose of this paper is to analyse a model for the field dynamics in a thin, essentially two-dimensional superconductor, and to show that it leads to vortex motion of the third type. The field equations are obtained from a Lagrangian whose kinetic terms are linear in the first time derivatives of the fields and whose potential part is the usual GinzburgLandau energy. This Lagrangian and its associated field equations are given in section 2. The Lagrangian is Galilean invariant, so we can see precisely how vortices respond to a transport current.

Section 3 is a review of the static vortex solutions of the Ginzburg-Landau theory, focussing especially on the manifold $M^{n}$, the $2 n$-dimensional parameter space of static $n$-vortex solutions which exist at the critical coupling $\lambda=1$. These solutions are also present in the model considered here, at special values of the couplings.

Section 4 treats the case where $\lambda$ is close to 1 , and where $n$-vortex motion can be well approximated by a motion on $M^{n}$. This adiabatic approximation assumes that at each instant the field is a static solution, but that the parameters of the static solution (i.e. the vortex positions) slowly vary with time. It is shown how the kinetic energy and potential energy of the field Lagrangian can be reduced to give a finite-dimensional dynamical system on $M^{n}$. The kinetic energy can be expressed in terms of local data associated with each of the vortices, and although an explicit form for this is not obtained, some conclusions can be drawn. In section 5 the equations of vortex motion are derived from the reduced Lagrangian, and it is shown that two vortices circle around one other.

The method used here for reducing the field dynamics to a particle dynamics for vortices

closely follows the analysis of slow vortex motion in the Abelian Higgs model with $\lambda=1$, as carried out by Samols [11]. There the reduced system's kinetic energy expression involves a Riemannian metric on $M^{n}$, the potential energy is a constant, and the vortex dynamics is modelled by geodesic motion on $M^{n}$ [12]. The geodesic motion is modified by the effect of a potential energy varying over $M^{n}$ if $\lambda$ is not exactly unity. This was studied by Shah [13]. Our reduced system has the same potential energy, and a kinetic energy which involves a connection or gauge potential on $M^{n}$ that depends on the same data as Samols' metric.

\section{The Schrödinger-Chern-Simons Lagrangian}

Let us consider a two-dimensional superconductor, with translational symmetry. A thin film with no defects might be close to this idealization. There is a complex scalar field $\phi$, representing the electron-pair condensate, and a gauge potential with time component 
$a_{t}$ and spatial components $a_{i}: i=1,2$. The magnetic field is $B=\partial_{1} a_{2}-\partial_{2} a_{1}$ and the electric field $E_{i}=\partial_{i} a_{t}-\dot{a}_{i}$. (An overdot denotes $\frac{\partial}{\partial t}$.) Units are fixed so that $\phi$ has magnitude 1 in the condensed phase (vacuum), and its covariant time and space derivatives are $D_{t} \phi=\partial_{t} \phi-i a_{t} \phi$ and $D_{i} \phi=\partial_{i} \phi-i a_{i} \phi$.

The Lagrangian that we shall consider, $L$, is an expression involving no higher than the first power of time derivatives. Explicitly,

$$
\begin{array}{r}
L=\int\left(\gamma \frac{i}{2}\left(\phi^{*} D_{t} \phi-\phi\left(D_{t} \phi\right)^{*}\right)+\mu\left(B a_{t}+E_{2} a_{1}-E_{1} a_{2}\right)-\gamma a_{t}\right. \\
\left.-\frac{1}{2} B^{2}-\frac{1}{2}\left(D_{i} \phi\right)^{*} D_{i} \phi-\frac{\lambda}{8}\left(1-|\phi|^{2}\right)^{2}-a_{i} J_{i}^{T}\right) d^{2} x,
\end{array}
$$

where $\frac{1}{2} B^{2}+\frac{1}{2}\left(D_{i} \phi\right)^{*} D_{i} \phi+\frac{\lambda}{8}\left(1-|\phi|^{2}\right)^{2}$ is the standard Ginzburg-Landau field energy density for static fields. Since there is no relativistic invariance, the summation convention is used only in the two space dimensions. The term with coefficient $\mu$ is the ChernSimons density for the gauge field. $\gamma, \mu$ and $\lambda$ are real constants, with $\lambda$ positive. $J_{i}^{T}$ is the transport current, that is, a constant vector in the plane of the superconductor. This Lagrangian is hardly original. The scalar field part of the Lagrangian coupled to the Chern-Simons term has appeared in the theory of Chern-Simons vortices, developed by Jackiw, Pi and others [14]. The inclusion of the $\gamma a_{t}$ term was advocated by Barashenkov and Harin to allow the possibility of a condensate $(|\phi|=1)$ at infinity [15]. Somewhat original is that the $\frac{1}{2} B^{2}$ term is included, but not the $\frac{1}{2} E_{i} E_{i}$ term usually present in the Maxwell Lagrangian. This is justified because there is no relativistic invariance in the rest of the Lagrangian, and it is desired to have only first time derivatives in the field equations. The field equations are

$$
\begin{aligned}
i \gamma D_{t} \phi & =-\frac{1}{2} D_{i} D_{i} \phi-\frac{\lambda}{4}\left(1-|\phi|^{2}\right) \phi \\
\epsilon_{i j} \partial_{j} B & =J_{i}^{S}-J_{i}^{T}+2 \mu \epsilon_{i j} E_{j} \\
2 \mu B & =\gamma\left(1-|\phi|^{2}\right)
\end{aligned}
$$

obtained by varying with respect to $\phi^{*}, a_{i}$ and $a_{t}$ respectively. $J_{i}^{S}$ is the supercurrent, defined by

$$
J_{i}^{S}=-\frac{i}{2}\left(\phi^{*} D_{i} \phi-\phi\left(D_{i} \phi\right)^{*}\right)
$$

Eq.(2.2) is the gauged non-linear Schrödinger equation, eq.(2.3) is Ampère's law, a twodimensional version of $\nabla \times \mathbf{B}=\mathbf{J}$, and eq.(2.4) is a constraint. There are really two 
contributions to the total current, namely, the supercurrent $J_{i}^{S}$ and the normal current $J_{i}^{N}$ which is present only in the cores of the vortices. This being a non-dissipative model, there is no Ohmic conductivity. However, there is a Hall conductivity $2 \mu$, and hence $J_{i}^{N}=$ $2 \mu \epsilon_{i j} E_{j}$. The effect of a Hall conductivity on vortex motion was previously considered by Dorsey [16], and equations (2.2)-(2.4) are just a special case of those analysed by Dorsey.

It is possible to have a constant, asymptotic value for the supercurrent $J_{i}^{S}$. This asymptotic current is called the transport current $J_{i}^{T}$. An everywhere constant current arises if $\phi=$ $\exp i\left(\mathbf{k} \cdot \mathbf{x}-|\mathbf{k}|^{2} t / 2 \gamma\right)$, for example, and then $\mathbf{J}^{T}=\mathbf{k}$. Such a constant current in a thin film produces a magnetic field above and below the film $\left(\frac{1}{2} \mathbf{k} \times \hat{\mathbf{z}}\right.$ above and $-\frac{1}{2} \mathbf{k} \times \hat{\mathbf{z}}$ below $)$ but no field in the film, and the three-dimensional Ampère's law is thereby satisfied. The version of Ampère's law (2.3) leaves out $\partial / \partial x_{3}$ terms. To correct for this and avoid a linear growth of $B$ in the plane, due to the constant current, it is necessary to subtract off $\mathbf{J}^{T}$ from $\mathbf{J}^{S}$, as in (2.3). This also explains the need for the $-a_{i} J_{i}^{T}$ term in the Lagrangian density.

The non-linear Schrödinger equation and its complex conjugate imply the supercurrent conservation law

$$
\partial_{i} J_{i}^{S}+\frac{\partial}{\partial t}\left(\gamma|\phi|^{2}\right)=0
$$

In addition, there is Faraday's law

$$
\partial_{1} E_{2}-\partial_{2} E_{1}+\frac{\partial B}{\partial t}=0
$$

which is an immediate consequence of the definitions of $E_{i}$ and $B$ in terms of the gauge potential. (The equation $\nabla \cdot \mathbf{B}=0$ is trivially satisfied in the two-dimensional geometry.) Taking the divergence of (2.3), and combining (2.6) and (2.7), one finds

$$
\frac{\partial}{\partial t}\left(2 \mu B+\gamma|\phi|^{2}\right)=0
$$

To avoid explicitly breaking translational invariance, $2 \mu B+\gamma|\phi|^{2}$ must be a constant, independent of position; to admit the condensate $|\phi|=1$ with no magnetic field, this constant must be $\gamma$. Hence $2 \mu B=\gamma\left(1-|\phi|^{2}\right)$, which agrees with (2.4).

An interpretation of eq.(2.4) is that the total electric charge density is zero. The charge density due to the condensate is $-|\phi|^{2}$, and the charge density of the background positive ions is 1 . Where the condensate is absent, the background positive charge is neutralised by decoherent, normal electrons; the normal charge density is $-\frac{2 \mu}{\gamma} B$. However, this is only 
an approximation, and the electric charge density is not exactly zero. Note that there is no equation for $\partial_{i} E_{i}$ among our field equations; this is because of the absence of $\frac{1}{2} E_{i} E_{i}$ in the Lagrangian. One may evaluate the charge density $\rho$ using $\partial_{i} E_{i}=\rho$; it is expected to be very small. Since Ampère's law requires that $\partial_{i} J_{i}^{\text {total }}=0$, one cannot use the current conservation equation of electrodynamics to deduce anything about $\rho$. Physically, there will be small electric charges generated by moving vortices, and $\partial_{i} J_{i}^{\text {total }}$ will not be exactly zero, but this can be ignored in the non-relativistic limit.

A remarkable property of the system of equations (2.2)-(2.4), together with (2.6) and (2.7), is that they are exactly Galilean invariant. It was stressed by Aitchison et al. that the equation for the scalar field should be Galilean invariant [10]. Our additional equations define a non-relativistic limit of Maxwell's equations with the same invariance. In fact, we have a "magnetic version" of Galilean invariance, with substantial currents and negligible electric charge density. For an illuminating discussion of Galilean invariant limits of electromagnetism, see ref. [17].

The basic Galilean transformation is a boost by a velocity v. Gauge invariant scalar quantities transform as $f \rightarrow f^{\prime}$ where $f^{\prime}(\mathbf{x}, t)=f(\mathbf{x}-\mathbf{v} t, t)$. Let us denote $\mathbf{x}^{\prime}=\mathbf{x}-\mathbf{v} t$. The field transformations are

$$
\begin{aligned}
& \phi^{\prime}(\mathbf{x}, t)=\phi\left(\mathbf{x}^{\prime}, t\right) e^{i \gamma\left(\mathbf{v} \cdot \mathbf{x}-\frac{1}{2}|\mathbf{v}|^{2} t\right)} \\
& a_{i}^{\prime}(\mathbf{x}, t)=a_{i}\left(\mathbf{x}^{\prime}, t\right) \\
& a_{t}^{\prime}(\mathbf{x}, t)=a_{t}\left(\mathbf{x}^{\prime}, t\right)-v_{i} a_{i}\left(\mathbf{x}^{\prime}, t\right) .
\end{aligned}
$$

It is straightforward to verify that eq.(2.2) is invariant under these transformations, in the same way that the Schrödinger equation in quantum mechanics is Galilean invariant when there is no potential. The magnetic and electric fields transform to

$$
\begin{aligned}
& B^{\prime}(\mathbf{x}, t)=B\left(\mathbf{x}^{\prime}, t\right) \\
& E_{i}^{\prime}(\mathbf{x}, t)=E_{i}\left(\mathbf{x}^{\prime}, t\right)-\epsilon_{i j} v_{j} B\left(\mathbf{x}^{\prime}, t\right)
\end{aligned}
$$

and the supercurrent becomes

$$
\mathbf{J}^{\prime S}(\mathbf{x}, t)=\mathbf{J}^{S}\left(\mathbf{x}^{\prime}, t\right)+\gamma \mathbf{v}\left|\phi\left(\mathbf{x}^{\prime}, t\right)\right|^{2}
$$

The transport current has therefore also transformed to

$$
\mathbf{J}^{\prime T}=\mathbf{J}^{T}+\gamma \mathbf{v}
$$


Combining eqs.(2.12)-(2.15), we find that eq.(2.3) is Galilean invariant provided the constraint (2.4) is satisfied. Finally, it is easily checked that the constraint (2.4) itself remains unchanged by a Galilean transformation.

The physical interpretation of the Galilean invariance is as follows. Given any solution of eqs.(2.2)-(2.4) in the absence of a transport current, the effect of a transport current $\mathbf{J}^{T}$ is simply to boost the solution so that it drifts along with the current at a velocity $\mathbf{v}=\frac{1}{\gamma} \mathbf{J}^{T}$. Since we now understand the effect of a transport current, let us from now on assume there isn't one, and that the fields have finite energy, with all currents and electromagnetic fields localized in a finite region of space.

\section{Vortices}

In the Ginzburg-Landau theory, fields which are smooth and of finite energy have the asymptotic behaviour $|\phi| \rightarrow 1$ and $D_{i} \phi \rightarrow 0$ at spatial infinity. Such fields are classified by their integer winding number. When the winding number is $n$, the phase of $\phi$ increases by $2 \pi n$ anticlockwise around the circle at infinity. The vanishing of $D_{i} \phi$ at infinity implies that the gauge potential also carries information about the winding number, from which follows the magnetic flux quantization

$$
\int B d^{2} x=2 \pi n
$$

If the zeros of $\phi$ are isolated, then the net number of zeros, counted with their multiplicity, is also $n$. A zero of multiplicity 1 may be identified as a magnetic flux vortex, and a zero of multiplicity -1 as an antivortex, so the winding number is also the net vortex number. The static Ginzburg-Landau equations

$$
\begin{gathered}
D_{i} D_{i} \phi+\frac{\lambda}{2}\left(1-|\phi|^{2}\right) \phi=0 \\
\epsilon_{i j} \partial_{j} B=J_{i}^{S}
\end{gathered}
$$

have a vortex solution with unit winding number of the form (in polar coordinates)

$$
\begin{gathered}
\phi=k(r) e^{i \theta} \\
a_{1}=-\frac{g(r)}{r} \sin \theta, \quad a_{2}=\frac{g(r)}{r} \cos \theta
\end{gathered}
$$

where $k \rightarrow 1$ and $g \rightarrow 1$ exponentially fast as $r \rightarrow \infty$, and where $k$ and $g$ both vanish at $r=0$. The precise form of $k$ and $g$ must be determined numerically. More generally, for 
winding number $n>1$, there are multivortex static solutions of a similar form, but with $\phi=k(r) e^{i n \theta}$, and where $k \rightarrow 1$ and $g \rightarrow n$ as $r \rightarrow \infty$. If $\lambda>1$, vortices repel, so the circularly symmetric multivortex solution is unstable to break-up into individual vortices. In the special case $\lambda=1$, there are no static forces between vortices. As Bogomolny showed [18], in this case eqs.(3.2) and (3.3) are satisfied, and the energy minimised, provided the first order Bogomolny equations

$$
\begin{gathered}
D_{1} \phi+i D_{2} \phi=0 \\
B=\frac{1}{2}\left(1-|\phi|^{2}\right)
\end{gathered}
$$

are satisfied. Eq.(3.5) only permits $\phi$ to have zeros of positive multiplicity, so solutions consist only of vortices. (To get antivortices, the sign in (3.5) and the sign of $B$ should be reversed.) The Bogomolny equations have not only circularly symmetric solutions, but, for any $n \geq 1$, solutions with vortices located at $n$ arbitrary points in the plane. More precisely, Taubes proved that, modulo gauge transformations, there is a unique finite energy solution of eqs.(3.5) and (3.6) with $\phi$ having zeros at any $n$ prescribed points $\left\{\mathbf{x}^{r}: 1 \leq r \leq n\right\}$, some of which may coincide [19]. Each such solution has winding $n$, total flux $2 \pi n$, and energy $\pi n$.

The parameter space of such solutions, which is called the $n$-vortex moduli space $M^{n}$, is a smooth manifold of dimension $2 n$. To see this, identify $\mathbb{R}^{2}$ and $\mathbb{C}$, and regard the zeros of $\phi$ as the complex numbers $\left\{z_{r}: 1 \leq r \leq n\right\}$. Geometrically, $M^{n}$ is the manifold $\mathbb{C}^{n} / \Sigma^{n}$ where $\Sigma^{n}$ is the permutation group acting on the $n$ zeros of $\phi . \mathbb{C}^{n} / \Sigma^{n}$ is actually smooth despite the apparent conical singularities where two or more zeros of $\phi$ coincide. The zeros uniquely define a polynomial $P(z)=z^{n}+a_{1} z^{n-1}+\ldots+a_{n}$ with precisely these zeros. The coefficients $\left\{a_{1}, \ldots, a_{n}\right\}$, which are symmetric polynomials in the zeros, are arbitrary complex numbers, so, as a manifold, $M^{n}$ is also the space of monic (leading coefficient $=1$ ) polynomials of degree $n$, and this is simply $\mathbb{C}^{n}$. The coefficients $\left\{a_{1}, \ldots, a_{n}\right\}$ rather than the unordered zeros $\left\{z_{1}, \ldots, z_{n}\right\}$ are the "good" coordinates on $\mathbb{C}^{n} / \Sigma^{n}$.

The equations (2.2)-(2.4) that we are interested in are not simply the static GinzburgLandau equations. However, if $\lambda=1$ and $\mu=\gamma$, any solution of the Bogomolny equations (3.5)-(3.6) is also a static solution of eqs.(2.2)-(2.4). The constraint (2.4) is one of the Bogomolny equations, so it is satisfied. $D_{t} \phi$ and $E_{i}$ can be consistently set to zero, with $a_{t}=0$.

One might seek static solutions of eqs.(2.2)-(2.4) for $\lambda \neq 1$. Presumably such solutions exist, if $a_{t}$ is allowed to be non-zero. They represent stationary points of the Ginzburg- 
Landau energy, subject to the constraint (2.4), with $a_{t}$ a Lagrange multiplier field. Solutions of the Bogomolny equations will come close to being solutions if $\lambda \simeq 1$ and $\mu \simeq \gamma$. As in the earlier case, with $\lambda \neq 1$, only circularly symmetric static solutions are expected, and they may again be unstable.

The constraint (2.4) is very far from being satisfied by the static solutions of the GinzburgLandau equations in the extreme Type II regime $(\lambda \gg 1)$. It is therefore unclear whether our model is of any relevance to vortex dynamics in this regime. Instead, we shall consider the case of $\lambda \simeq 1$, which is realised by niobium and vanadium in certain temperature ranges.

\section{A Reduced Lagrangian}

The rest of this paper is devoted to constructing a reduced Lagrangian for $n$-vortex dynamics, assuming $\lambda \simeq 1$. We need to assume that $\mu=\gamma$; this is essential for simplifying the kinetic energy. The method is similar to that used to study $n$-vortex dynamics in the Abelian Higgs model, both at $\lambda=1$ and when $\lambda \simeq 1$. Let us consider fields which at each instant are static solutions of the Bogomolny equations, but where the moduli, that is, the vortex positions $\mathbf{x}^{r}$ (or better, the coefficients of the polynomial $P(z)$ ) are time-dependent. These moduli will vary slowly if $\lambda$ is close to 1 . The fields are inserted in the Lagrangian (2.1), and the integrals carried out, where possible. The result is a reduced Lagrangian for motion on the moduli space $M^{n}$. The reduced system is an approximation to the true field dynamics, but we shall not try to estimate the errors involved. The use of solutions of the Bogomolny equations is possibly better justified here than in the context of the Abelian Higgs model, because the constraint (2.4), which is one of the Bogomolny equations, must be satisfied.

Let us denote by $\left\{X^{\alpha}: 1 \leq \alpha \leq 2 n\right\}$ some general coordinates on the moduli space $M^{n}$, for example, the components of the vortex positions. The field Lagrangian has a kinetic term which is first order in time derivatives, and a potential term. The reduced system is therefore expected to have a Lagrangian of the form

$$
L=\mathcal{A}_{\alpha}(\mathbf{X}) \dot{X}^{\alpha}-V(\mathbf{X}) .
$$

$L$ may have additional terms which are total time derivatives, but these do not affect the dynamics. $\mathcal{A}_{\alpha}$ has the interpretation of a gauge potential or connection on $M^{n}$, and it is somewhat arbitrary since replacing it by $\mathcal{A}_{\alpha}^{\prime}=\mathcal{A}_{\alpha}+\partial_{\alpha} \Lambda$ (a gauge transformation) changes $L$ by the total time derivative $\frac{d \Lambda}{d t}$. From $L$ we find the equations of motion

$$
\mathcal{B}_{\alpha \beta} \dot{X}^{\alpha}+\partial_{\beta} V=0
$$


where $\mathcal{B}_{\alpha \beta}=\partial_{\alpha} \mathcal{A}_{\beta}-\partial_{\beta} \mathcal{A}_{\alpha}$ is the curvature of the connection $\mathcal{A}$. The motion according to (4.2) is non-dissipative, with $V$ constant along any solution path $X^{\alpha}(t)$.

The potential energy in the reduced model is the Ginzburg-Landau potential energy, but because the fields satisfy the Bogomolny equations, this simplifies to [18]

$$
V=n \pi+\frac{\lambda-1}{8} \int\left(1-|\phi|^{2}\right)^{2} d^{2} x
$$

The constant $n \pi$ does not affect the dynamics. The integral without the factor $\frac{1}{8}(\lambda-1)$ is positive, and is some function of the relative positions of the vortices, invariant under a rigid rotation. The detailed form of the integral is not known, for general $n$, but it is expected to be minimal when the vortices are well separated, and maximal when the vortices are coincident. The integral has been computed in the case of two vortices, by Shah [13]. It increases monotonically as the vortex separation decreases.

We can proceed much further with the calculation of the kinetic energy in the reduced model. Let us start with the kinetic terms of the field Lagrangian, with $\gamma=\mu$

$$
T=\gamma \int\left(\frac{i}{2}\left(\phi^{*} \dot{\phi}-\phi \dot{\phi}^{*}\right)+|\phi|^{2} a_{t}-a_{t}+B a_{t}+\left(\partial_{2} a_{t}-\dot{a}_{2}\right) a_{1}-\left(\partial_{1} a_{t}-\dot{a}_{1}\right) a_{2}\right) d^{2} x
$$

It helps to make some assumptions about the asymptotic gauge. Recall that vortices are exponentially localized. Gauge invariant quantities $B,\left|D_{i} \phi\right|$ and $|\phi|$ approach their asymptotic values exponentially fast. Suppose that the solutions of the Bogomolny equations are in the gauge where, for large $r$, they are of the form $\phi=e^{i n \theta}, a_{1}=-\frac{n}{r} \sin \theta, a_{2}=\frac{n}{r} \cos \theta$, with at most exponentially small corrections. Since $E_{i}$ and $D_{t} \phi$ are exponentially small asymptotically, in this gauge $a_{t}$ is exponentially small too.

Using the appropriate version of Stokes' theorem, the terms involving derivatives of $a_{t}$ can now be removed, and $T$ expressed as

$$
T=\gamma \int\left(\frac{i}{2}\left(\phi^{*} \dot{\phi}-\phi \dot{\phi}^{*}\right)+a_{2} \dot{a}_{1}-a_{1} \dot{a}_{2}\right) d^{2} x+\gamma \int\left(|\phi|^{2}-1+2 B\right) a_{t} d^{2} x
$$

The boundary terms vanish as a result of the gauge fixing, and the Bogomolny equation (3.6) (or the constraint (2.4)) implies that the second integral vanishes. Now define $a=$ $a_{1}+i a_{2}$. Then $T$ reduces to the compact expression

$$
T=-\gamma \int \operatorname{Im}\left(\phi^{*} \dot{\phi}+a^{*} \dot{a}\right) d^{2} x
$$


Let us next express the scalar field as

$$
\phi=e^{\frac{1}{2} h+i \chi}
$$

where $h$ and $\chi$ are real. $\phi$ is a smooth function, but the gauge is not fixed except asymptotically, so there is considerable freedom in the choice of $\chi$. Because of the presence of vortices, $\chi$ is multivalued, increasing by an integer multiple of $2 \pi$ along an anticlockwise loop around any zero of $\phi$. However, in a given gauge, the gradient and time derivative of $\chi$ are well-defined.

Away from the zeros of $\phi$, the Bogomolny equation (3.5) implies that

$$
a=\frac{1}{2} \partial_{2} h+\partial_{1} \chi+i\left(-\frac{1}{2} \partial_{1} h+\partial_{2} \chi\right)
$$

and hence

$$
\dot{a}=\frac{1}{2} \partial_{2} \dot{h}+\partial_{1} \dot{\chi}+i\left(-\frac{1}{2} \partial_{1} \dot{h}+\partial_{2} \dot{\chi}\right) .
$$

Also

$$
\dot{\phi}=\left(\frac{1}{2} \dot{h}+i \dot{\chi}\right) e^{\frac{1}{2} h+i \chi}
$$

Therefore

$$
T=-\gamma \int\left(e^{h} \dot{\chi}+\left(\frac{1}{2} \partial_{2} h+\partial_{1} \chi\right)\left(-\frac{1}{2} \partial_{1} \dot{h}+\partial_{2} \dot{\chi}\right)-\left(-\frac{1}{2} \partial_{1} h+\partial_{2} \chi\right)\left(\frac{1}{2} \partial_{2} \dot{h}+\partial_{1} \dot{\chi}\right)\right) d^{2} x .
$$

It is easy to verify that (4.11) can be reexpressed as

$$
T=-\gamma \int\left(\left(e^{h}-\nabla^{2} h\right) \dot{\chi}-\frac{1}{2} \frac{\partial}{\partial t}(\nabla h \cdot \nabla \chi)+\partial_{2} f_{1}-\partial_{1} f_{2}\right) d^{2} x
$$

where

$$
f_{1}=\left(\partial_{2} h+\partial_{1} \chi\right) \dot{\chi}+\frac{1}{4} \partial_{1} h \dot{h}, \quad f_{2}=\left(-\partial_{1} h+\partial_{2} \chi\right) \dot{\chi}+\frac{1}{4} \partial_{2} h \dot{h} .
$$

Since, from (4.8), $B=-\frac{1}{2} \nabla^{2} h$, the Bogomolny equation (3.6) reduces to

$$
\nabla^{2} h-e^{h}+1=0,
$$

so $T$ simplifies to

$$
T=-\gamma \int\left(\dot{\chi}-\frac{1}{2} \frac{\partial}{\partial t}(\nabla h \cdot \nabla \chi)+\partial_{2} f_{1}-\partial_{1} f_{2}\right) d^{2} x
$$


To progress, we need to specify carefully the region of integration. It will simplify our calculations to assume that at any time $t$, the vortex positions $\mathbf{x}^{r}(t)$ are distinct, so $\phi$ has a simple zero at each of these $n$ points. The case of two or more of these points becoming coincident can be treated by taking a limit. The fields $\phi$ and $a$ are smooth functions of space and time, so the original expression (4.4) for $T$ has no singularities. However, $h$ and $\chi$ are ill-defined at the (moving) vortex locations, and $\nabla h, \nabla \chi, \dot{h}$ and $\dot{\chi}$ all diverge as a vortex is approached. Let us therefore define the region of integration $\Sigma$ to be that obtained from $\mathbb{R}^{2}$ by removing $n$ discs of small radius $\epsilon$, centred at the vortex locations $\mathbf{x}^{r}(t)$. This produces an error of order $\epsilon^{2}$ in $T$. At the end of the calculation, let $\epsilon \rightarrow 0$. During the calculation we can neglect any terms in $T$ (but not in the component fields) that are $O(\epsilon)$ or smaller.

Let $C^{r}(\epsilon)$ denote the circular boundary of the $r$ th disc. The terms involving $f_{1}$ and $f_{2}$ reduce to line integrals along $C^{r}(\epsilon)$. There is no contribution from infinity as $f_{1}$ and $f_{2}$ decay exponentially fast. The integral of $\frac{\partial}{\partial t}(\nabla h \cdot \nabla \chi)$ can be expressed in terms of a total time derivative. We have

$$
\frac{d}{d t} \int_{\Sigma} \nabla h \cdot \nabla \chi d^{2} x=\int_{\Sigma} \frac{\partial}{\partial t}(\nabla h \cdot \nabla \chi) d^{2} x-\sum_{r=1}^{n} \int_{C^{r}(\epsilon)}(\nabla h \cdot \nabla \chi) \dot{\mathbf{x}}^{r} \times \mathbf{d} \mathbf{l}
$$

taking into account that the boundary $C^{r}(\epsilon)$ is moving with velocity $\dot{\mathbf{x}}^{r}$. The line element $\mathbf{d l}$ is that along $C^{r}(\epsilon)$, and $\dot{\mathbf{x}}^{r} \times \mathbf{d l}$ is a scalar (in two dimensions). If we drop the total time derivative from $T$, which does not affect the dynamics, the integral of $\frac{\partial}{\partial t}(\nabla h \cdot \nabla \chi)$ is simply replaced by integrals over the circles $C^{r}(\epsilon)$.

Combining these observations we find

$$
T=-\gamma \int_{\Sigma} \dot{\chi} d^{2} x+\frac{\gamma}{2} \sum_{r=1}^{n} \int_{C^{r}(\epsilon)}(\nabla h \cdot \nabla \chi) \dot{\mathbf{x}}^{r} \times \mathbf{d l}-\gamma \sum_{r=1}^{n} \int_{C^{r}(\epsilon)} \mathbf{f} \cdot \mathbf{d} \mathbf{l}
$$

To calculate the integrals along $C^{r}(\epsilon)$ we need the expansions of $h$ and $\chi$ around the vortex centre $\mathbf{x}^{r}$. Since $\phi$ is linear in the neighbourhood of $\mathbf{x}^{r}$, to first approximation, $h$ has the expansion [11]

$$
h=\log \left|\mathbf{x}-\mathbf{x}^{r}(t)\right|^{2}+\alpha^{r}+\beta_{1}^{r}\left(x_{1}-x_{1}^{r}(t)\right)+\beta_{2}^{r}\left(x_{2}-x_{2}^{r}(t)\right)+\ldots
$$

where $\alpha^{r}, \beta_{1}^{r}$ and $\beta_{2}^{r}$ depend on the locations of the other vortices, and vary smoothly with time as the vortices move. The contours of $|\phi|$ are approximately circles near $\mathbf{x}^{r}$, 
and $\left(\beta_{1}^{r}, \beta_{2}^{r}\right)$ measures the extent to which the centres of these circles differ from $\mathbf{x}^{r}$ as $|\phi|$ increases, because of the other vortices.

From (4.18), we find that near $\mathbf{x}^{r}$

$$
\nabla h=\left(\frac{2\left(x_{1}-x_{1}^{r}\right)}{\left|\mathbf{x}-\mathbf{x}^{r}\right|^{2}}+\beta_{1}^{r}, \frac{2\left(x_{2}-x_{2}^{r}\right)}{\left|\mathbf{x}-\mathbf{x}^{r}\right|^{2}}+\beta_{2}^{r}\right)
$$

and

$\dot{h}=-\left(\frac{2\left(x_{1}-x_{1}^{r}\right)}{\left|\mathbf{x}-\mathbf{x}^{r}\right|^{2}}+\beta_{1}^{r}\right) \dot{x}_{1}^{r}-\left(\frac{2\left(x_{2}-x_{2}^{r}\right)}{\left|\mathbf{x}-\mathbf{x}^{r}\right|^{2}}+\beta_{2}^{r}\right) \dot{x}_{2}^{r}+\dot{\alpha}^{r}+\dot{\beta}_{1}^{r}\left(x_{1}-x_{1}^{r}\right)+\dot{\beta}_{2}^{r}\left(x_{2}-x_{2}^{r}\right)$

with higher order corrections that can be neglected. Let us introduce a polar angle $\theta^{r}$ relative to the (moving) vortex at $\mathbf{x}^{r}$, with $\theta^{r}=0$ in the positive $x_{1}$ direction. Then on $C^{r}(\epsilon)$

$$
\nabla h=\left(\frac{2 \cos \theta^{r}}{\epsilon}+\beta_{1}^{r}, \frac{2 \sin \theta^{r}}{\epsilon}+\beta_{2}^{r}\right)
$$

and

$$
\dot{h}=-\left(\frac{2 \cos \theta^{r}}{\epsilon}+\beta_{1}^{r}\right) \dot{x}_{1}^{r}-\left(\frac{2 \sin \theta^{r}}{\epsilon}+\beta_{2}^{r}\right) \dot{x}_{2}^{r}+\dot{\alpha}^{r}+\epsilon\left(\dot{\beta}_{1}^{r} \cos \theta^{r}+\dot{\beta}_{2}^{r} \sin \theta^{r}\right)
$$

Next, suppose that the gauge has been chosen so that on and inside the circle $C^{r}(\epsilon)$, the phase $\chi$ of the field $\phi$ is exactly linearly dependent on the polar angle $\theta^{r}$, that is

$$
\chi=\theta^{r}+\psi^{r}
$$

where $\psi^{r}$, which will be referred to as the orientation of vortex $r$, depends only on time. Then, on $C^{r}(\epsilon)$

$$
\nabla \chi=\left(-\frac{\sin \theta^{r}}{\epsilon}, \frac{\cos \theta^{r}}{\epsilon}\right)
$$

and

$$
\dot{\chi}=\frac{1}{\epsilon}\left(\dot{x}_{1}^{r} \sin \theta^{r}-\dot{x}_{2}^{r} \cos \theta^{r}\right)+\dot{\psi}^{r}
$$

We shall see that it is not possible globally to set $\psi^{r}=0$, although it would be on a short time interval. 
We can now evaluate the line integrals along $C^{r}(\epsilon)$ in the kinetic energy expression (4.17). Note that $\mathbf{d} \mathbf{l}=\epsilon\left(-\sin \theta^{r}, \cos \theta^{r}\right) d \theta^{r}$, and $\dot{\mathbf{x}}^{r} \times \mathbf{d} \mathbf{l}=\epsilon\left(\dot{x}_{1}^{r} \cos \theta^{r}+\dot{x}_{2}^{r} \sin \theta^{r}\right) d \theta^{r}$. Therefore

$$
\begin{aligned}
\int_{C^{r}(\epsilon)}(\nabla h \cdot \nabla \chi) \dot{\mathbf{x}}^{r} \times \mathbf{d} \mathbf{l} & =\int_{0}^{2 \pi}\left(-\beta_{1}^{r} \sin \theta^{r}+\beta_{2}^{r} \cos \theta^{r}\right)\left(\dot{x}_{1}^{r} \cos \theta^{r}+\dot{x}_{2}^{r} \sin \theta^{r}\right) d \theta^{r} \\
& =\pi\left(\beta_{2}^{r} \dot{x}_{1}^{r}-\beta_{1}^{r} \dot{x}_{2}^{r}\right)
\end{aligned}
$$

and

$$
\begin{aligned}
\int_{C^{r}(\epsilon)} \mathbf{f} \cdot \mathbf{d} \mathbf{l}= & -\int_{0}^{2 \pi}\left(1+\epsilon\left(\beta_{2}^{r} \sin \theta^{r}+\beta_{1}^{r} \cos \theta^{r}\right)\right)\left(\frac{1}{\epsilon}\left(\dot{x}_{1}^{r} \sin \theta^{r}-\dot{x}_{2}^{r} \cos \theta^{r}\right)+\dot{\psi}^{r}\right) d \theta^{r} \\
& \quad-\frac{1}{2} \int_{0}^{2 \pi}\left(-\beta_{1}^{r} \sin \theta^{r}+\beta_{2}^{r} \cos \theta^{r}\right)\left(\dot{x}_{1}^{r} \cos \theta^{r}+\dot{x}_{2}^{r} \sin \theta^{r}\right) d \theta^{r} \\
= & -2 \pi \dot{\psi}^{r}-\frac{3 \pi}{2}\left(\beta_{2}^{r} \dot{x}_{1}^{r}-\beta_{1}^{r} \dot{x}_{2}^{r}\right)
\end{aligned}
$$

so

$$
T=-\gamma \int_{\Sigma} \dot{\chi} d^{2} x+2 \pi \gamma \sum_{r=1}^{n}\left(\dot{\psi}^{r}+\left(\beta_{2}^{r} \dot{x}_{1}^{r}-\beta_{1}^{r} \dot{x}_{2}^{r}\right)\right)
$$

We still need to consider the integral of $\dot{\chi}$. This is not the time derivative of the integral of $\chi$, since $\chi$ is multivalued and its integral over the plane ill-defined. However, a gauge transformation replaces $\chi$ by $\chi+\tilde{\chi}$ where $\tilde{\chi}$ is single-valued, and the integral of $\dot{\chi}$ changes by the time derivative of the integral of $\tilde{\chi}$. So, up to a total time derivative, the integral of $\dot{\chi}$ is gauge invariant. It is convenient, in this integral, to extend the region of integration back to the whole plane. $\dot{\chi}$ is $O\left(\frac{1}{\epsilon}\right)$ near the vortices, so the contribution to its integral from the discs of radius $\epsilon$ is $O(\epsilon)$ and can be neglected.

The integral cannot be evaluated directly. Instead, consider its integral over a finite time interval

$$
\int_{t_{0}}^{t_{1}} \int_{\mathbb{R}^{2}} \dot{\chi} d^{2} x d t
$$

Suppose that the initial and final configurations are the same, that is, the vortex locations are the same, and the fields are in the same gauge. Consider first the simple case where just one vortex moves anticlockwise around a loop which does not enclose any other vortices. At a point outside the loop, $\chi$ varies but there is no net change in $\chi$ between the initial and final time. (At infinity this is true, and by continuity this result extends to any point outside the loop.) At a point inside the loop, on the other hand, $\chi$ increases by $2 \pi$. This can 
be verified by deforming the motion of the vortex around the point into an anticlockwise motion of the point around the vortex. The integral (4.29) is therefore $2 \pi$ times the area of the loop. For a more general closed vortex trajectory, possibly with self-crossings, the integral is $2 \pi$ times the signed area enclosed.

The result generalizes. Even if the loop encloses other non-moving vortices, the integral is $2 \pi$ times the area of the loop. If all $n$ vortices move, the integral is $2 \pi$ times the sum of the areas enclosed by the $n$ vortex loops. Finally, we must allow for the vortices to exchange locations. Suppose two vortices move anticlockwise along half-loops, such that they exchange places. Then the integral is $2 \pi$ times the area enclosed by the loop. All these results can be checked by using a specific model for $\chi$, for example the phase of the complex polynomial $P(z)=\prod_{r=1}^{n}\left(z-z_{r}(t)\right)$, where $z$ and $z_{r}(t)$ are the complex numbers representing a general point in the plane and the trajectory of the $r$ th vortex. Then it is easy to calculate the change in $\chi$ at $z$ due to the vortex motion. Unfortunately, this model doesn't quite satisfy our requirements on the phase at infinity or on the circles $C^{r}(\epsilon)$, but this can be dealt with easily.

Now observe that the correct value for the integral of $\dot{\chi}$ over space and time, as discussed above, can be obtained from the local expression

$$
\pi \sum_{r=1}^{n}\left(-x_{2}^{r} \dot{x}_{1}^{r}+x_{1}^{r} \dot{x}_{2}^{r}\right)
$$

whose integral over time gives again $2 \pi$ times the sum of the (signed) areas enclosed by the vortex trajectories. So the expression (4.30) is equal to the integral of $\dot{\chi}$ over the plane, up to an ignorable time derivative. Therefore $T$ can be rewritten as

$$
T=2 \pi \gamma \sum_{r=1}^{n}\left(\dot{\psi}^{r}+\left(\beta_{2}^{r}+\frac{1}{2} x_{2}^{r}\right) \dot{x}_{1}^{r}-\left(\beta_{1}^{r}+\frac{1}{2} x_{1}^{r}\right) \dot{x}_{2}^{r}\right),
$$

and this is our final expression for $T$. The term (4.30) has occurred before, in the context of ungauged vortices [20], although the neat calculation in [20] involves the manipulation of a divergent integral. For well separated vortices, the $\mathbf{x}^{r}$ terms in (4.31) dominate, since $\beta_{1}^{r}$ and $\beta_{2}^{r}$ are exponentially small. The coefficients $\beta_{1}^{r}$ and $\beta_{2}^{r}$ have a significant effect when the vortex cores are overlapping. Their appearance is not surprising as they also appear in the expression for the Riemannian metric on $M^{n}$, and Samols has determined some of their properties which will not all be used here, but which could be useful [11].

The $\dot{\psi}^{r}$ terms are topological. Along a closed path in $M^{n}$, with the initial and final configurations identical, the initial and final values of the orientations $\psi^{r}$ of the vortices 
are geometrically the same, but they may have been permuted, and they may also have changed by integer multiples of $2 \pi$. The motion of the vortices relative to each other defines a braid, and the integral over time of $\sum_{r=1}^{n} \dot{\psi}^{r}$ can depend only on this braid. In fact, each positive generator of the braid group contributes $2 \pi$ to the integral, as we shall see below.

There is an important consistency check on the expression (4.31) for $T$. Note that, as anticipated, $T$ defines a connection on the moduli space $M^{n}$. The integral of this connection around any closed loop on $M^{n}$ is called the holonomy around that loop. It can be calculated by integrating $T$ over time, for motion (at any speed) around the loop. Since $M^{n}$ has no singularities, the holonomy should vanish as a loop contracts to a point. In particular, consider the closed loop on $M^{2}$ where two vortices exchange places by moving anticlockwise along semi-circular trajectories. This motion is a generator of the braid group. The holonomy should become zero as the radius shrinks to zero. Let the vortex trajectories be $\mathbf{x}^{1}(t)=R(\cos t, \sin t)$ and $\mathbf{x}^{2}(t)=-R(\cos t, \sin t)$, with $0 \leq t \leq \pi$. The complex polynomial representation $P(z)=\left(z-z_{1}(t)\right)\left(z-z_{2}(t)\right)$ shows that the orientations of the two vortices are $\psi^{1}(t)=t$ and $\psi^{2}(t)=\pi+t$, so the integral of $\dot{\psi}^{1}+\dot{\psi}^{2}$ is $2 \pi$. Also, by circular and reflection symmetry, $\left(\beta_{1}^{1}, \beta_{2}^{1}\right)$ is of the form $\beta(R)(\cos t, \sin t)$ and $\left(\beta_{1}^{2}, \beta_{2}^{2}\right)=-\left(\beta_{1}^{1}, \beta_{2}^{1}\right)$. A simple calculation shows that the total holonomy is

$$
-2 \pi \gamma\left(\pi R^{2}-2 \pi+2 \pi \beta(R) R\right)
$$

The coefficient $\beta(R)$ is defined as that occurring in the expansion of $h$ around $\mathbf{x}^{1}=(R, 0)$ when the vortices are at $(R, 0)$ and $(-R, 0)$. By reflection symmetry, this expansion has the form

$$
h=\log \left(\left(x_{1}-R\right)^{2}+\left(x_{2}\right)^{2}\right)+\alpha(R)+\beta(R)\left(x_{1}-R\right)+\ldots
$$

For large $R$, the vortex at $(-R, 0)$ has an exponentially small effect, so $\beta(R)$ is exponentially small and the holonomy is proportional to $\pi R^{2}-2 \pi$, the $2 \pi$ being a topological correction to the area of the circle enclosed by the vortex trajectories. When $R$ is small, $h$ can be estimated simply from the leading logarithmic terms due to both vortices

$$
h=\log \left(\left(x_{1}-R\right)^{2}+\left(x_{2}\right)^{2}\right)+\log \left(\left(x_{1}+R\right)^{2}+\left(x_{2}\right)^{2}\right)+\ldots .
$$

Expanding about $(R, 0)$, this becomes

$$
h=\log \left(\left(x_{1}-R\right)^{2}+\left(x_{2}\right)^{2}\right)+\log 4 R^{2}+\frac{1}{R}\left(x_{1}-R\right)+\ldots
$$


so $\beta(R)=\frac{1}{R}$ for small $R$. This singular behaviour is just what is needed for the holonomy to vanish as $R \rightarrow 0$.

Presumably, a more sophisticated version of this argument would establish that the holonomy vanishes for any loop on $M^{n}$ as the loop shrinks to a point.

\section{Vortex Motion}

The Lagrangian derived in section 4 for the motion of $n$ vortices is

$$
L=2 \pi \gamma \sum_{r=1}^{n}\left(\dot{\psi}^{r}+\left(\beta_{2}^{r}+\frac{1}{2} x_{2}^{r}\right) \dot{x}_{1}^{r}-\left(\beta_{1}^{r}+\frac{1}{2} x_{1}^{r}\right) \dot{x}_{2}^{r}\right)-V\left(\mathbf{x}^{1}, \mathbf{x}^{2}, \ldots, \mathbf{x}^{n}\right)
$$

where $V$ is the integral expression (4.3). The term $\dot{\psi}^{r}$ is locally a time derivative, and so has no effect on the vortex motion, although it has a topological significance as we have seen. A rather similar Lagrangian has been obtained for well separated vortices by Dziarmaga [21], using Berry phase methods.

The general form of the equation of motion for vortex $r$ is

$$
\frac{d}{d t}\left(\frac{\partial L}{\partial \dot{x}_{i}^{r}}\right)-\frac{\partial L}{\partial x_{i}^{r}}=0
$$

For $L$ as above, this becomes

$$
\begin{aligned}
& 2 \pi \gamma\left[\dot{x}_{1}^{r}+\left(\frac{\partial \beta_{1}^{r}}{\partial x_{1}^{s}}+\frac{\partial \beta_{2}^{s}}{\partial x_{2}^{r}}\right) \dot{x}_{1}^{s}+\left(\frac{\partial \beta_{1}^{r}}{\partial x_{2}^{s}}-\frac{\partial \beta_{1}^{s}}{\partial x_{2}^{r}}\right) \dot{x}_{2}^{s}\right]-\frac{\partial V}{\partial x_{2}^{r}}=0 \\
& 2 \pi \gamma\left[\dot{x}_{2}^{r}+\left(\frac{\partial \beta_{2}^{r}}{\partial x_{2}^{s}}+\frac{\partial \beta_{1}^{s}}{\partial x_{1}^{r}}\right) \dot{x}_{2}^{s}+\left(\frac{\partial \beta_{2}^{r}}{\partial x_{1}^{s}}-\frac{\partial \beta_{2}^{s}}{\partial x_{1}^{r}}\right) \dot{x}_{1}^{s}\right]+\frac{\partial V}{\partial x_{1}^{r}}=0
\end{aligned}
$$

with summation over $s$ implied. It is straightforward to check that $V$ is constant along a trajectory. This implies that if $n$ vortices are initially well separated, they remain so, and similarly if they are initially all close together.

Symmetries imply further conservation laws. Consider the Lagrangian for motion on $M^{n}$ in the general form (4.1). If $\xi^{\alpha}(\mathbf{X})$ is the vector field generating a symmetry (i.e. the Lie derivatives $\mathcal{L}_{\xi} \mathcal{A}$ and $\mathcal{L}_{\xi} V$ vanish), then $\mathcal{A}_{\alpha}(\mathbf{X}) \xi^{\alpha}(\mathbf{X})$ is a constant of the motion. The symmetries here are translations and rotations in the plane. The associated conserved quantities that follow from the specific Lagrangian (5.1) are, respectively,

$$
\sum_{r=1}^{n}\left(\beta_{1}^{r}+\frac{1}{2} x_{1}^{r}\right), \sum_{r=1}^{n}\left(\beta_{2}^{r}+\frac{1}{2} x_{2}^{r}\right)
$$


and

$$
\sum_{r=1}^{n}\left(\left(\beta_{1}^{r}+\frac{1}{2} x_{1}^{r}\right) x_{1}^{r}+\left(\beta_{2}^{r}+\frac{1}{2} x_{2}^{r}\right) x_{2}^{r}\right) .
$$

Samols [11] has shown that for any locations of the vortices,

$$
\sum_{r=1}^{n} \beta_{1}^{r}=\sum_{r=1}^{n} \beta_{2}^{r}=0
$$

so the conservation of the quantities (5.4) implies that the centre of the $n$-vortex system, $\frac{1}{n} \sum_{r=1}^{n} \mathbf{x}^{r}$, is fixed.

If there is just one vortex, it does not move. This is because $\beta_{1}$ and $\beta_{2}$ vanish, by circular symmetry or by (5.6), and $V$ is independent of the vortex position.

Two vortices move around each other in a circular motion, rather like two fluid vortices of equal strength. To see this, change coordinates, writing

$$
\begin{aligned}
& \mathbf{x}^{1}=\mathbf{X}^{0}+R(\cos \theta, \sin \theta) \\
& \mathbf{x}^{2}=\mathbf{X}^{0}-R(\cos \theta, \sin \theta) .
\end{aligned}
$$

As was remarked in the previous section, $\left(\beta_{1}^{1}, \beta_{2}^{1}\right)=-\left(\beta_{1}^{2}, \beta_{2}^{2}\right)=\beta(R)(\cos \theta, \sin \theta)$. The potential $V$ depends only on $R$. In these coordinates, the Lagrangian simplifies to

$$
L=2 \pi \gamma\left(2 \dot{\theta}+X_{2}^{0} \dot{X}_{1}^{0}-X_{1}^{0} \dot{X}_{2}^{0}-R(R+2 \beta(R)) \dot{\theta}\right)-V(R)
$$

The term $2 \dot{\theta}$ equals $\dot{\psi}^{1}+\dot{\psi}^{2}$. It has been included, although it does not affect the equations of motion, which are

$$
\begin{gathered}
\dot{X}_{1}^{0}=\dot{X}_{2}^{0}=0, \dot{R}=0 \\
2 \pi \gamma\left(\frac{d}{d R}\left(R^{2}+2 R \beta(R)\right)\right) \dot{\theta}=-\frac{d V}{d R} .
\end{gathered}
$$

The centre $\mathbf{X}^{0}$ does not move, and the relative motion is at constant angular velocity on a fixed circle.

At large separation, $\frac{d V}{d R}$ and $\beta$ are exponentially small, so $\dot{\theta}$ is exponentially small. The maximum angular velocity occurs at a finite separation of order the vortex core size. At small separation, $\frac{d V}{d R} \simeq O\left(R^{3}\right)$. This is because $V$ has a maximum at $R=0$, and varies quadratically with the "good" radial coordinate on $M^{2}$, which is $R^{2}$. The $\frac{1}{R}$ singularity 
in $\beta(R)$ does not produce a singular coefficient of $\dot{\theta}$, so the angular velocity vanishes as $R$ tends to zero. In the limit, the two vortices are coincident and at rest. It would be a useful consistency check to see if there is a corresponding exact, static solution of the field equations.

Symmetry implies that if there are $p$ vortices at the vertices of a regular $p$-gon, and $q$ vortices at the centre (possibly with $q=0$ ), then the $p$-gon will rigidly rotate with its centre fixed.

\section{Conclusions}

In this paper, a time-dependent Ginzburg-Landau model for a complex scalar field coupled to electromagnetism in two space dimensions has been considered. The Lagrangian incorporates the standard potential energy of Ginzburg-Landau theory, and there is a Schrödinger kinetic term for the scalar field and a Chern-Simons term for the electromagnetic field. The model is exactly Galilean invariant, so fields respond to a transport current simply by a velocity boost parallel to the current. The model has vortex solutions, and at the critical coupling $\lambda=1$, it has the $2 n$-dimensional manifold $M^{n}$ of static $n$-vortex solutions satisfying the Bogomolny equations. The parameters of these solutions are the positions of the $n$ zeros of the scalar field, which can be identified with the vortex positions.

If $\lambda \neq 1$, and there are two or more vortices, then they generally move, but without dissipation. For $\lambda$ close to 1 , the field dynamics describing vortex motion can be approximated by considering solutions of the Bogomolny equations with time-varying parameters. This leads to a reduced Lagrangian for motion on $M^{n}$, with a kinetic term linear in the vortex velocities, and a potential term. Remarkably, the kinetic term depends only on local data associated with each vortex. It defines a gauge potential on $M^{n}$ which depends smoothly on the vortex positions, even as the vortices become coincident.

For two vortices, the gradient of the potential energy is along the line joining them, but the motion is at right angles to this, and they orbit each other at constant separation. It would be interesting to study the motion of more than two vortices in this model. This will require more detailed computations of the potential energy and the gauge potential on $M^{n}$, or a numerical simulation of the field dynamics.

The quantisation of the vortex motion should also be considered.

\section{Acknowledgements}

I am grateful to several people for discussions about this work, including N. Goldenfeld, D. 
Thouless, G. Volovik, A. Gill, and J. Dziarmaga. I am especially indebted to I. Aitchison for his DAMTP seminar on the time-dependent Ginzburg-Landau theory, and to G. Gibbons for discussions about Galilean invariance.

This work is partly supported by EPSRC grant GR/K50641, part of the Applied Nonlinear Mathematics Programme. 


\section{References}

1. C.P. POOLE JR., H.A. FARACH AND R.J. CRESWICK, "Superconductivity", Academic Press, San Diego, 1995;

D.R. TILLEY AND J. TILLEY, "Superfluidity and Superconductivity (3rd ed.)", Inst. of Phys. Publishing, Bristol, 1990.

2. A. ORAL, S.J. BENDING AND M. HENINI, Appl. Phys. Lett. 69 (1996) 1324.

3. A.A. ABRIKOSOV, Sov. Phys. JETP 5 (1957) 1174.

4. L. JACOBS AND C. REBBI, Phys. Rev. B19 (1979) 4486.

5. H.B. NIELSEN AND P. OLESEN, Nucl. Phys. B61 (1973) 45.

6. A. VILENKIN AND E.P.S. SHELLARD, "Cosmic Strings and other Topological Defects", Cambridge U.P., Cambridge, 1994.

7. L.P. GOR'KOV AND G.M. ÉLIASHBERG, Sov. Phys. JETP 27 (1968) 328;

S.J. CHAPMAN, S.D. HOWISON AND J.R. OCKENDON, SIAM Rev. 34 (1992) 529.

8. S. DEMOULINI AND D. STUART, "Gradient flow of the superconducting GinzburgLandau functional on a plane", to appear in Commun. Anal. and Geom.;

D. STUART, Appl. Math. Lett. 9 (1996) 27.

9. P.G. DE GENNES, "Superconductivity in Metals and Alloys", Benjamin, New York, 1966; P. NOZIÈRES AND W.F. VINEN, Philos. Mag. 14 (1966) 667.

10. I.J.R. AITCHISON, P. AO, D.L. THOULESS AND X.-M. ZHU, Phys. Rev. B51 (1995) 6531 ;

M. STONE, Int. J. Mod. Phys. B9 (1995) 1359.

11. T.M. SAMOLS, Commun. Math. Phys. 145 (1992) 149.

12. N.S. MANTON, Phys. Lett. B110 (1982) 54.

13. P.A. SHAH, Nucl. Phys. B429 (1994) 259.

14. R. JACKIW AND S.-Y. PI, Phys.Rev. D42 (1990) 3500.

15. I.V. BARASHENKOV AND A.O. HARIN, Phys. Rev. Lett. 72 (1994) 1575.

16. A.T. DORSEY, Phys Rev. B46 (1992) 8376. 
17. M. LE BELLAC AND J.-M. LÉVY-LEBLOND, Nuovo Cim. 14B (1973) 217.

18. E.B. BOGOMOLNY, Sov. J. Nucl. Phys. 24 (1976) 449.

19. A. JAFFE AND C. TAUBES, "Vortices and Monopoles", Birkhäuser, Boston, 1980;

C. TAUBES, Commun. Math. Phys. 72 (1980) 277.

20. Q. LIU AND A. STERN, Phys. Rev. D52 (1995) 1300.

21. J. DZIARMAGA, Phys. Rev. B53 (1996) 8231. 